\title{
Constitución y modelo social europeo
}

\author{
ENRIQUE BARÓN CRESPO \\ Catedrático Jean Monnet "ad personam" \\ e-mail: enrique.baron@european-foundation.org
}

\section{RESUMEN}

El presente artículo pretende contextualizar, conceptualizar y cuantificar la construcción de la Europa Social. Para ello, se aborda la puesta en marcha y evolución del modelo social europeo, se realiza un balance económico y se apuntan algunas ideas sobre el futuro de dicho modelo.

Palabras clave: Espacio social europeo, construcción europea, futuro espacio social.

\section{Constitution and European Social Model}

\begin{abstract}
This article aims to contextualize, conceptualize and quantify the construction of the Social Europe. To do so, it addresses the implementation and evolution of the European social model; it valuates economic balance and suggests some ideas about the future of this model.

Keywords: Social European Space, European Construction, The Future of the European Social Space.

Clasificación JEL: F15.
\end{abstract}

Artículo disponible en versión electrónica en la página www.revista-eea.net, ref. ə-27310. 
Estudiar la construcción de la Europa social para hacer un balance económico es un ambicioso proyecto que requiere una delimitación conceptual previa sobre el concepto Europa social. En efecto, si se trata de estudiar la Europa monetaria, la consideración se centrará en la Unión monetaria con el Euro como valor básico y central, mientras que hablar de lo social es multifacético, puede abarcar todos los aspectos de la vida en sociedad. Caben distintos enfoques: la dimensión social como complemento de la económica, considerar que se trata de una cuestión de diálogo social clásico en el marco de las relaciones industriales, o un modelo social consagrado en la norma y gestionado a nivel europeo. En cierto modo, lo social se parece al aire o al amor en que es más fácil sentirlo que describirlo o teorizarlo.

Desde esta perspectiva, es un elemento normalmente aceptado a la hora de comparar los sistemas económicos que el europeo se caracteriza por la existencia de una economía social de mercado, en la que los principios del Estado del bienestar, recogidos en el informe Beveridge ${ }^{1}$ definen una sociedad de la que el estadounidense Jeremy Rifkin dice que su sueño es que "todos queden incluidos y no se deje a nadie en la cuneta". Afirmación que adquiere todo su valor en la actual situación de crisis global, si se considera que tanto el Presidente Obama en EE.UU. como el Partido Comunista Chino consideran la universalización de la asistencia sanitaria como un elemento esencial de respuesta a la crisis además de su valor de justicia social.

Por tanto, las dos cuestiones básicas a responder son: la existencia de un modelo social europeo y su cuantificación.

\section{¿EXISTE UN MODELO SOCIAL EUROPEO?}

Los padres fundadores compartían algunos principios fundamentales de lo querían construir, no tanto desde el punto de vista teórico como de la experiencia vivida de las dos grandes guerras civiles europeas del Siglo XX. Se pueden resumir en dos: crear sistemas con valores comunes de la democracia y el respeto de los derechos humanos y superar el desarrollo del capitalismo en el marco del Estado Nación con la lucha por la hegemonía continental.

El método elegido fue el paso a paso con la creación de la CECA y después, el Mercado Común. En su gestación, la dimensión social estuvo presente desde sus comienzos. El testimonio de los redactores del Tratado de Roma es más ilustrativo que muchos comentarios sin base al respecto. Los plumíferos fueron Pierre Uri, un filósofo francés judío y de Hans von der Groeben, un alto funcionario alemán colaborador del régimen nazi. No cabe ejemplo más gráfico de lo que fue la reconciliación europea. Lo mejor es recoger sus testimonios: para Uri, "no se podía seguir exactamente el modelo de la CECA, donde prácticamente se conocía cada empresa

\footnotetext{
${ }^{1}$ Beveridge, "Report to the Parliament on Social Insurance and Allied Services", House of Commons, nov. 1942.
} 
y las dificultades de cada cuenca. Era preciso hacer algo gradual, que tuviera en cuenta a la vez las realidades de nuestro tiempo, es decir, construir un sistema en el que una integración no condujera al mismo desequilibrio que la unificación italiana que enriqueció al Norte y empobreció al Sur, en el que se tendrían en cuenta los problemas sociales: se fijarían las reglas de la competencia, se crearía una política de empleo con un fondo social y una política regional con una banca de inversiones"2. Por su parte, Von der Groeben explica que "conseguimos lograr un acuerdo sobre todos los problemas, lo cual es muy interesante porque Uri era un poco el padre de la CECA y sus ideas fueron introducidas en el Tratado, concepción en mi opinión indefendible y prácticamente inviable. Uri comprendió muy bien que con esta concepción muy dirigista no era posible hacer un mercado común con los alemanes y los holandeses. En cuanto a Italia, no sabía muy bien cual era su concepción económica. Luigi Einaudi y los liberales estaban ciertamente de acuerdo con Erhard (entonces Ministro de Economía)y conmigo, pero había otras opiniones más dirigistas. La concepción del Mercado Común es una concepción del tipo "Soziale Marktwirschaft", no es una economía dirigista. Uri y yo nos pusimos de acuerdo sobre esta concepción"3.

En esencia, se trata de las cuatro libertades y en especial el establecimiento y circulación de las personas, así como en la de los servicios. Basta con viajar por el resto del mundo para comprender lo que se ha conseguido en la eliminación de barreras aduaneras, controles burocráticos y limitaciones a la libertad personal. Con dos componentes más, que no se suelen asociar pero que forman parte del núcleo duro de competencias exclusivas europeas: la política de competencia, con su dimensión de lucha antimonopolio y la política comercial exterior común, poderosa arma europea como primera potencia comercial.

En la práctica, la realización del mercado común a partir de la unión aduanera llenó la primera parte de la historia de la construcción europea desde sus inicios hasta el final de la guerra fría. Tras la inicial tarea de reconstrucción, la acción se concentró en eliminar barreras físicas y posiciones de poder económico para garantizar las normas de funcionamiento de la economía de mercado en un marco supranacional.

Al mismo tiempo se iban construyendo en paralelo los Estados Miembros de la CE como Estados del bienestar. El "holandés Van der Beugel, también negociador del Tratado de Roma, dice al respecto: "lo que no podíamos prever, era la existencia del Estado del bienestar que no existía en 1955. Y el Estado del bienestar es un animal muy nacional. La legitimidad del Estado Nación fue muy fortalecida por el Estado del bienestar. La gente mira hacia su capital cuando se trata de su jubilación o su seguridad social, no hacia Bruselas ${ }^{4}$. El proceso general en la Europa Occidental de la posguerra fue la configuración de los países como Estados sociales de

\footnotetext{
${ }^{2}$ Vid. "La génése des Traités de Rome. Entretiens inédits avec 18 acteurs et témoins de la négotiation" Fondation Jean Monnet pour l'Europe. Editions Economica. Paris, 2007, p. 259.

${ }^{3}$ La génése, op. cit., p. 118.

${ }^{4}$ La génése, op.cit., p. 351.
} 
Derecho sobre la base de sistemas de economía capitalista mixta, cuya fórmula se puede resumir en Estado del bienestar + keynesianismo + pacto fruto del diálogo social. Se trataba de la respuesta democrática al fascismo y al comunismo que permitió la incorporación activa de la mayoría de la población en el sistema político, (sufragio universal con incorporación del voto femenino, trabajadores y campesinos) y gracias al sistema centralizado e igualitario, previsión y cobertura de riesgos (maternidad, enfermedad, accidente de trabajo, paro, fallecimiento...) desde la cuna hasta la cuna hasta la tumba para el conjunto de la población.

De ser una reivindicación histórica del movimiento obrero y la socialdemocracia o la democracia cristiana social este sistema se ha convertido en una parte integral del consenso social básico, lo cual hace muy difícil su desmantelamiento (por ejemplo, por el thatcherismo). Además, si se tiene en cuenta su arraigo entre los ciudadanos, con variantes que forman parte de la historia y la idiosincrasia de cada país, es un tema de muy difícil trasferencia.

En la segunda fase de activa de la construcción europea en la década de 1980, momento en el que España se incorporó de modo activo a la construcción europea, la Comisión Delors planteó la incorporación de la dimensión social al mercado interior (actualización del mercado común que se pacto estaría acabado en 1970), con un refuerzo de las políticas estructurales (regional y social), completado con el lanzamiento de la Unión Económica y Monetaria sobre la base de la moneda. única, en una proceso que preveía el salto político con el nuevo siglo.

En 1989, la histórica conmoción producida por la caída del muro, símbolo de un cambio de época (implosión del bloque soviético y fin de la guerra fría), junto a la aceleración de la globalización con la incorporación de potencias emergentes, el progreso tecnológico, los cambios demográficos, el cambio climático $\mathrm{y}$, sobre todo, la mundialización de la aspiración a la democracia) dieron un impulso sustancial al proceso.

La respuesta fue el Tratado de la Unión Europea de Maastricht con el salto de la Comunidad a la Unión Europea, completada con la ciudadanía, la moneda única y la incorporación de la cohesión (económica, territorial y social) y la creación de un sistema de pilares para incluir la política exterior y de seguridad así como la de interior y justicia. La Carta Social sólo se aprobó entre 11 Estados por la oposición conservadora británica. La Gran Bretaña solo la firmó en 1997, tras la victoria de Blair.

Se abre con ello la segunda fase de la construcción europea, configurada como una Unión Política, Económica y Monetaria de dimensión supranacional y ciudadanos. Construcción original, formada por Estados Nación enfrentados durante siglos y pionera, que en tres oleadas de ampliación ha pasado en una década de componerse de 12 Estados a 27 con más negociaciones de adhesión en curso.

La agenda, de centrarse en lo económico y comercial, pasa a ser fundamentalmente política, concretada con ritmo cuatrienal en sucesivos Tratados (Amsterdam, Niza, dos Convenciones que produjeron la Carta de Derechos Fundamentales y la Constitución, y su rescate por el Tratado de Lisboa en vías de ratificación). Dos cuestiones se plantean como fundamentales: la primera es la introducción con éxito 
del Euro como moneda única, lo cual implica la aceptación de un valor social básico compartido por todos los ciudadanos, además de un profundo cambio cultural. La gente no tiene que cambiar moneda para comparar. Además, se plantea la necesidad de proseguir con ritmo en la construcción europea, porque no existen precedentes de Unión Monetaria a largo plazo sin avance paralelo de Unión Política. Para comprender la importancia de este paso, basta con imaginar lo que hubiera ocurrido en la actual crisis financiera global sin moneda única: los especuladores hubieran jugado a la pelota con nuestras divisas respectivas, como ha recordado el ex Canciller Helmut Schmidt.

La segunda es la construcción de la Europa social. La inclusión de la ciudadanía en el Tratado de Maastricht, infravalorada en su momento, ha tenido efectos expansivos continuos. Ante todo, ha configurado el espacio europeo de libertad, seguridad y justicia, cuya expresión más directa es Schengen, con la eliminación de controles fronterizos interiores (con excepción de Gran Bretaña e Irlanda) y se desarrolló en la primera Convención que produjo la Carta de Derechos Fundamentales, con participación de Estados (Gobiernos y Parlamentos) además del Parlamento Europeo y la Comisión, La Estrategia de Lisboa, primera decisión comunitaria que reunió y sistematizó los objetivos socioeconómicos de la Unión (empleo, $\mathrm{I}+\mathrm{D}$, formación y cohesión) completó la formulación.

La Constitución, rescatada en el Tratado de Lisboa en su contenido con evidente pérdida de claridad en la forma, contiene por primera vez, las líneas fundamentales del modelo social europeo en sus tres primeros artículos: la ciudadanía, la no discriminación, la solidaridad y la igualdad entre mujeres y hombres, la protección de los derechos del niños y la solidaridad intergeneracional .Define como objetivos de la UE una economía social de mercado altamente competitivas tendente al pleno empleo y al progreso social, el combate de la exclusión social y la discriminación, el fomento de la justicia, la protección social y la cohesión económica, social y territorial, así como la necesidad de adoptar medidas de garantía en los servicios económicos de interés general.

Hay dos componentes con efectos políticos y jurisprudenciales importantes: el primero es la Carta de Derechos Fundamentales, proclamada solemnemente y con valor vinculante en el Tratado de Lisboa y una "cláusula social horizontal" en virtud de la cual la Unión debe tener en cuenta, para elaborar sus políticas, las exigencias relacionadas con un alto nivel de empleo, la garantía de la protección social adecuada, la lucha contra la exclusión social, la educación, la formación y la protección de la salud.

La coordinación de las políticas económicas y de creación de empleo fue objeto de uno de los enfrentamientos mayores en el seno de la Convención. El resultado es que la UE puede adoptar medidas para garantizar la coordinación de las políticas económicas, en particular en relación con las orientaciones generales y adopción de directrices en política de empleo y se reconoce la especificidad de la zona Euro aunque no se cree la figura del Ministro de Economía y se mantiene la unanimidad en la fiscalidad incluso en la relacionada con el mercado interior. 
Con ello, las funciones de la UE en relación con el modelo social, además de las declarativas, consistentes en proclamar principios, valores y derechos que deben inspirar la acción comunitaria, son las funciones protectoras, reguladoras y redistribuidoras.

- Funciones protectoras, campo donde la labor del tribunal de Justicia es pionera al ir afirmando derechos humanos en el trabajo como derechos personales (dignidad, igualdad, libertad). De este modo, la no discriminación y acoso en el puesto de trabajo, la igualdad entre sexos y la legitimación para recurrir por parte de los sindicados y movimientos sociales han adquirido carta de naturaleza. Filosofía innovadora de los derechos sociales más avanzada que las legislaciones de la mayor parte de los Estados miembros y supone un nuevo enfoque al no se tener en cuenta sólo los derechos colectivos propios del capitalismo industrial tipo "fordista" sino que incorpora la dimensión personal del ciudadano en cuanto tal.

- Funciones reguladoras. con normas relativas a la seguridad e higiene en el puesto de trabajo o los derechos de ciudadanos europeos que viven en países distintos al suyo de origen que por su misma naturaleza no han sido demasiado polémicas, mientras que la reglamentación en temas como permiso de maternidad, derechos de información y participación de los trabajadores, trabajo temporal, horario máximo de trabajo o libertad de prestación de servicios han sido mucho más dificultosos no sólo por resistencias ideológicas sino también por la misma concepción de los sistemas de relaciones industriales, Hay estados en donde el Derecho del Trabajo responde a una tradición reglamentista con un sindicalismo reivindicativo y débil (Francia) mientras que en otros la estructura responde mucho más a un sistema negociador con un fuerte peso de la negociación colectiva (Italia, Alemania o Suecia) El desarrollo de la vía de diálogo y negociación en el marco europeo entre los interlocutores sociales es una vía importante de avance.

- Funciones redistribuidoras. No se puede pensar en una gestión centralizada del modelo social y del bienestar en la UE, con un Presupuesto comunitario que se eleva a un $1 \%$ de la Renta Interna Bruta conjunta (1,27\% de techo actual y 1,07 previsible si se aprueba las perspectivas financieras) frente al 30$50 \%$ de los Estados miembros. Además de la aplicación de los principios de subsidiariedad y proporcionalidad.

Además de los textos de los Tratados, está la vida política, con el permanente desafío de la construcción de mayorías sociales, que si es complicado a nivel estatal, lo es mucho más en una esfera pública con una opinión naciente como es la europea. Así, en la última legislatura europea, han tenido enorme importancia política y económica batallas como las desarrolladas en la gestación y aprobación en codecisión (entre Consejo y Parlamento Europeo) de las directivas de servicios, tiempo de trabajo, trabajadores desplazados o permiso de maternidad. Igualmente, 
la tendencia jurisprudencial del Tribunal de Justicia de Luxemburgo en casos como Viking y Laval, es de mínimos más que de máximos. Aunque reconoce el derecho de huelga, establece que no prevalece sobre las libertades de establecimiento de las empresas ni de prestación de servicios.

\section{BALANCES ECONÓMICOS}

Sobre su avance, se han hecho diversos balances económicos. Un informe esencial por su repercusión fue el famoso Informe Mac Dougall ${ }^{5}$, encargado por la Comisión Europea a un grupo de expertos, sobre "El papel de las Finanzas públicas en la integración europea" en 1977. Partía de la siguiente consideración inicial: "Es posible concebir, presumiblemente en alguna lejana fecha, una federación en Europa en la cual el gasto público federal se sitúe en torno al 20-25\% del PIB, como en EE.UU. o en Alemania. Un paso previo sería una federación con un gasto federal más reducido del orden del 5 al $7 \%$ o del 7 al $10 \%$ si no se incluyera la defensa. Una característica esencial de dicha federación sería que la prestación de servicios sociales y de bienestar quedaría en su casi totalidad a escala nacional". Conviene señalar que Sir Donald Mac Dougall no era un enfebrecido eurofederalista sino el Jefe de la Asesoría Económica de la CBI, la patronal británica.

Pasados más de 30 años, la propuesta sigue siendo revolucionaria, ya que el Presupuesto de la UE que llegó al 1,27\% en la etapa de Maastricht no llega al 1\% actualmente, con Unión Económica y Monetaria. No obstante, las propuestas del informe influyeron en la elaboración de las políticas regional y social. Un nuevo informe Mac Dougall II, que encargué como Presidente del Parlamento Europeo de cara a la negociación de las perspectivas financieras con el Tratado de Maastricht sobre una nueva estrategia para la cohesión económica y social después de 1992" 6 , señalaba ya los límites y las disfunciones de un enfoque meramente cuantitativo y ponía el acento en su dimensión cualitativa a través de opciones estratégicas en educación e investigación, con inclusión, en los criterios de atribución de ayudas, de indicadores de calidad de vida y bienestar de los ciudadanos, conducentes a luchar prioritariamente contra la pobreza en lugar de limitarse al enfoque macroeconómico.

El Informe Cecchini sobre "El coste de la No Europa", fue otro destacado estudio para calcular las ventajas de la culminación del mercado interior en el Desafío $92^{7}$. El método utilizado fue calcular los ahorros que produciría la realización del mercado interior, reuniendo trabajos de prestigiosos institutos. En concreto, las ganancias en el lado de la oferta derivadas de la eliminación de obstáculos al comercio (fronteras, trámites administrativos y financieros) se estimaban entre 8 a 9

\footnotetext{
5 Mac Dougall "Report on the role of public finance in European integration", 1977 $\mathrm{http} / / / \mathrm{ec}$.europa.eu/economy_finance/emu_history/documentation, pp. 13-4.

${ }^{6}$ National Institute of Economic and Social Research, London, 1992.

7 “1992, le Défi”. Flammarion, con prólogo de Jacques Delors.
} 
millardos de ECUS; las derivadas de la eliminación de obstáculos a la producción (reserva de mercados públicos objeto de concurso, reglas técnicas, obstáculos a la circulación de capitales y servicios y al transporte, entre 57 y 71 millardos de ECUS. En la demanda, los consumidores se beneficiarían de una rebaja de precios medios del $21 \%$ en productos como hipotecas, seguros, créditos al consumo o equipos de telecomunicación. Sumando todo ello, con las economías de escala correspondiente más las ganancias de productividad se podía alcanzar un total de 200.000 millardos de ECUS. La perspectiva era un crecimiento suplementario del $4,5 \%$, una baja del nivel de precios del $6 \%$ así como una reducción en las perspectivas presupuestarias de los Estados miembros. La argumentación fue valiosa para movilizar un esfuerzo colectivo que permitió aprobar el paquete de 300 directivas que culminó el mercado interior en 1992 y que dio pasó a la implementación de la Unión Económica y Monetaria, preparada por el Comité Delors, con el resultado de la creación exitosa del $€$.

En la actualidad, la presentación anual por la Comisión al Parlamento del Informe sobre la cohesión económica y social se ha convertido en una tradición. En el Sexto Informe, de junio de 2009, se hace un análisis en profundidad de la creatividad y la innovación como elementos clave para salir de la crisis. Se trata de estudios valiosos sobre todo en el terreno de la cohesión territorial en donde la experiencia y los resultados de la política europea son pioneros, con casos tan espectaculares como Irlanda o España, lo cual no modifica el hecho básico de que las políticas sociales se siguen haciendo por los Estados miembros.

Estos ejemplos, planteados dentro de las competencias de la Unión Europea muestran la enorme complejidad del balance económico de su dimensión social, más aún si se tiene presente que en este tema las opciones políticas juegan un papel importante. Así, como se ha puesto de manifiesto en la elaboración y adopción de la directiva de servicios, una de las directivas más controvertidas de la anterior legislatura, objeto de un demagógico debate en el referéndum francés sobre la Constitución, (el famoso caso del fontanero polaco), es evidente que la valoración de los servicios económicos de interés general no es la misma por parte de un conservador o de un socialdemócrata como tampoco la concepción de la política de educación o sanidad entre un francés y un alemán.

\section{EL FUTURO DEL MODELO SOCIAL}

No resulta fácil hacer un pronóstico sobre el modelo social europeo que no tenga en cuenta la visión del mundo de su autor. No obstante, se puede señalar que la cuestión estará cada vez más en el centro de la escena europea, definida a partir del mercado interior más la ciudadanía europea y la moneda única. La entrada en vigor del Tratado de Lisboa supondrá la afirmación, por primera vez de forma explícita de principios, valores y objetivos que configuran una concepción de la sociedad. Es de esperar que contribuyan positivamente a configurar un marco en el que se 
desarrolle un debate democrático a nivel europeo entre las principales familias políticas además de los Estados.

Además, no se trata de un debate doméstico. En este comienzo de legislatura, la gran pregunta que plantea la cargada agenda global del G 20, es si la Unión Europea es capaz de estar a la altura de los desafíos planteados: salir de la crisis y revivir la economía creando empleo y avance tecnológico, reorganizar el sistema financiero global y recuperar la confianza, concluir la ronda de Doha para favorecer el comercio internacional y conseguir un acuerdo en Copenhague para frenar al cambio climático.

El Presidente de la Comisión, José Manuel Durão Barroso, al presentar ante el Parlamento Europeo sus "Orientaciones políticas para la próxima Comisión”, se ha formulado en voz alta la pregunta ¿Queremos ser líderes, modelando la globalización sobre la base de nuestros valores e intereses, o dejaremos que otros tomen la iniciativa y aceptaremos los resultados que estos modelen?, en claro contraste con la línea neoliberal y un tanto "pasotista" de su primer mandato. En gran medida, el futuro del modelo social europeo depende de nuestra capacidad de incluir esta cuestión en la agenda global del G 20 y, sobre todo, de la ONU. 
